\title{
RUDJER BOŠKOVIĆ INSTITUTE RADIOCARBON MEASUREMENTS XI
}

\author{
DUŠAN SRDOČ, BOGOMIL OBELIĆ, NADA HORVATINČIĆ \\ INES KRAJCAR BRONIĆ \\ Rudjer Bošković Institute, P O Box 1016, 41001 Zagreb, Yugoslavia
}

and

\author{
ADELA SLIEPČEVIĆ \\ Faculty of Veterinary Medicine, University of Zagreb
}

\section{INTRODUCTION}

This is a list of radiocarbon analyses determined since our previous list ( $R, 1987, v$ 29, no. 1, p 135-147). As before, with the exception of speleothems and groundwaters, the calculations follow conventional RADIOCARBON protocol (Stuiver \& Polach, 1977). Sample pretreatment, combustion and counting techniques are essentially the same as described in R, 1971, v 13, no. 1, p 135-140, supplemented by new techniques for groundwater processing (R, 1979, v 21, no. 1, p 131-137). Speleothem and groundwater model ages (given in the comment section of each listing) are based on an initial activity of $85 \pm 10$ percent modern carbon (pMC), as recommended in Srdoč et al (1986), without adjustment for $\delta^{13} \mathrm{C}$. These model ages can be converted from the 5570-yr half-life to the chronometrically more correct half-life of $5730 \mathrm{yr}$ by multiplying by the factor 1.029. The " \pm figures" on these model ages are statistical combinations of the $1 \sigma$ counting uncertainty and the $\pm 10 \%$ uncertainty in the assumed 85 pMC. Ages and " \pm figures" of all other samples are adjusted for stable isotope fractionation according to the recommendations in Stuiver and Polach (1977). Data processing is done by computer (Obelić \& Planinić, 1977; Obelić, 1980). Sample descriptions are prepared in collaboration with collectors and submitters.

\section{ACKNOWLEDGMENTS}

We thank E Hernaus for preparation of samples and methane synthesis, A Turković for data processing and P Hojski for technical assistance.

\section{ARCHAEOLOGIC SAMPLES}

\section{Z-1641. Vladikina ploča cave}

$290 \pm 100$

Wood fragment (Quercus sp) from soil wall in Vladikina ploča cave, Rsovac near Pirot $\left(43^{\circ} 11^{\prime} \mathrm{N}, 22^{\circ} 45^{\prime} \mathrm{E}, 715 \mathrm{~m}\right.$ asl) $15 \mathrm{~m}$ from entrance, $1.5 \mathrm{~m}$ above ground. Coll and subm by D Gavrilović, Univ Belgrade. Comment (DG): expected age: $500 \mathrm{BP}$. Dates period of cave occupation.

\section{Z-1674. Pepelane}

$5850 \pm 130$

Charcoal from prehistoric layer, depth $1.6 \mathrm{~m}$ at Pepelane near Virovitica $\left(45^{\circ} 44^{\prime} \mathrm{N}, 17^{\circ} 30^{\prime} \mathrm{E}\right) \mathrm{E}$ Slavonia, Croatia. Coll and subm 1985 by K Minichreiter, Regional Inst Preservation Cultural Monuments, Osijek. Comment (KM): expected age: 4 th millennium BC. 


\section{Z-1752. Koprivnički bregi}

Charcoal underlying plowed humus, $25 \mathrm{~cm}$ thick, pit S-III/IV, at Koprivnički bregi-Seče $\left(46^{\circ} 10^{\prime} \mathrm{N}, 14^{\circ} 34^{\prime} \mathrm{E}\right), 124 \mathrm{~m}$ asl. Coll and subm 1984 by Z Marković, Town Mus Koprivnica. Comment (ZM): expected age: 5000-6000 вP.

\section{Z-1862. Okrugla pećina}

$98.6 \pm 1.4$ pMC

Charcoal from Okrugla pećina cave at Gornje Sinjevo near Sarajevo, Bosnia. Coll and subm 1986 by DJ Basler, Regional Mus, Sarajevo. Comment: (DJB): expected age: Bronze Age. Possible contamination with recent material.

\section{Z-1742. Vela spilja}

$5430 \pm 100$

Charcoal mixed with clay from fireplace in Vela spilja cave, 2.70 to $2.90 \mathrm{~m}$ below ground level, near Vela Luka, Korčula I, Croatia. Coll and subm 1986 by B Čečuk, Archaeol Inst Yugoslav Acad Sci \& Arts, Zagreb. Comment (BČ): expected age: $5000 \mathrm{BP}$.

\section{Ajdovska jama cave series}

Charcoal and speleothems from Ajdovska jama cave, site of systematic excavation of Neolithic settlement near Nemška Vas, Krško, E Slovenia. Coll and subm 1986 by A Šercelj and M Horvat, Slov Acad Sci \& Arts, Ljubljana. Dates correspond to earlier measurements, Z-1042 to-1045 (R, 1984, v 26, no. 3, p 451) and Z-1178, -1179, -1554, -1602, -1603 (R, 1987, v 29, no. 1, p 138-139).

\section{Z-1822. Ajdovska jama No. 25A}

$6900 \pm 130$

Speleothem from central hall, depth $2.2 \mathrm{~m}$.

Z-1860. Ajdovska jama No. 33

$5420 \pm 150$

Charcoal from central hall, depth $2.17 \mathrm{~m}$. Sample assoc with human and animal bones.

\section{Z-1875. Zagreb skeletons}

$100.0 \pm 1.4 \mathrm{pMC}$

Bone collagen from human skeletons discovered during reconstruction of city sewage, Zagreb $\left(45^{\circ} 48^{\prime} \mathrm{N}, 16^{\circ}, 0^{\prime} \mathrm{E}\right)$ Croatia. Coll and subm 1987 by D Strinović, Inst Forensic Med, Med Fac, Zagreb. Comment (DS): expected age: Middle Ages to Modern.

Tar from pit, $60 \mathrm{~cm}$ deep, in prehistoric site at Sigetec near Ludbreg $\left(46^{\circ} 16^{\prime} \mathrm{N}, 16^{\circ} 38^{\prime} \mathrm{E}\right) \mathrm{NW}$ Croatia. Coll and subm 1980 by M Šimek, Town Mus Varaždin.

Timber fragments from wall under fresco paintings from medieval church Sv Lovro dated in 13th or 14th century AD in Slavonska Požega $\left(45^{\circ}\right.$ 
$20^{\prime} \mathrm{N}, 17^{\circ} 41^{\prime}$ E) Slavonia, Croatia. Coll and subm 1987 by J Velnić, Croatian Inst Restoration, Zagreb.

\section{Vučedol series}

Continuation of excavations at Eneolithic site "Streim's vineyard," on bank of Danube R near Vukovar ( $\left.45^{\circ} 21^{\prime} \mathrm{N}, 19^{\circ} 01^{\prime} \mathrm{E}\right) 110 \mathrm{~m}$ asl, E Croatia. Most finds were coll from oval pits used as waste dumps and burying grounds. Samples coll and subm 1986 and 1987 by A Durman, Dept Archaeol, Univ Zagreb. Dated to determine span of Baden, Kostolac, and Vučedol cultural layers (R, 1987, v 29, no. 1, p 135-136: Horvatinčić et al, in press).

Z-1820. Vučedol

$4370 \pm 140$

Charcoal, Pit 32, Kostolac phase.

Z-1821. Vučedol

$4500 \pm 150$

Charcoal, Pit 103, Kostolac phase.

Z-1863. Vučedol

$4190 \pm 180$

Collagen from human bones, Pit 13, Vučedol phase.

Z-1864. Vučedol

$4620 \pm 150$

Collagen from human bones, Pit 2, Baden phase.

\section{Z-1865. Vučedol}

$4030 \pm 140$

Collagen from human bones, Pit 6 , Vučedol phase.

General Comment (AD): results correspond to second Indo-European migration wave (Gimbutas, 1980) and place Vučedol settlement in same period as Ezero, Sitagroi IV-V, Poliochni II-III, and Troy I-II.

\section{Vinkovci series}

Two charcoal samples from Eneolithic site near Vinkovci $\left(45^{\circ} 16^{\prime} \mathrm{N}\right.$, $18^{\circ} 49^{\prime} \mathrm{E}$ ) ca $20 \mathrm{~km} \mathrm{~W}$ of Vučedol; dated to determine duration of Vučedol culture in this area. Coll and subm 1986 by A Durman.

\section{Z-1817. Vinkovci 1}

Charcoal, Pit VH 175, depth 3.4m. Comment (AD): expected age: Vučedol phase.

Z-1818. Vinkovci 2

$3830 \pm 140$

Charcoal, House HV 226, depth 1.5m. Comment (AD): expected age: Vinkovci phase.

General Comment: disturbed strata at Vinkovci site consist of Vučedol and Vinkovci-Somogyvár cultures.

\section{Liber Linteus Zagrabiensis series}

Coll 1848 by M Barić and subm 1986 by I Mirnik and A RendićMiočević, Archaeol Mus Zagreb. Comment: dated to determine true age of 
Etruscan linen book with long inscription which found its way to Egypt to be used as mummy wrappings. Besides linen cloth, other datable materials, such as embalming unguent and leaves assoc with mummy were dated. Samples were sent to Groningen for interlaboratory comparison of data (Srdoč \& Horvatinčić, 1986, in press).

Z-1653. Mummy linen wrappings $2290 \pm 80$

Comment: GrN-13875: $2210 \pm 13,2335 \pm 15$.

Z-1682. Embalming unguent $2230 \pm 90$

Fraction insoluble in $\mathrm{CHCl}_{3}$; $\mathrm{GrN}-13860: 2240 \pm 13$.

Z-1654. Leaves (Dicotyledon)

$$
2580 \pm 90
$$

Comment: very limited amount of leaves resulted in considerable error and deviation from mean values of linen and unguent age; GrN-14467: $2825 \pm 15$.

\section{Z-1675. Embalming unguent}

$4540 \pm 120$

Exuded fraction. Comment $(\mathrm{NH})$ : much older age of exuded fraction indicates that several components used in preparation of unguent contained carbon much older than rest of components.

\section{Z-1496. Egyptian sarcophagus}

$2700 \pm 110$

Wood from Egyptian sarcophagus of private collection. Subm 1985 by E Kavurić, Zagreb. Calibrated date corresponds to 22nd dynasty.

\section{GEOLOGIC SAMPLES}

Fossil Wood

\section{Z-1881. Trenta valley}

$6820 \pm 120$

Fossil wood (unid. sp) buried in lake chalk, Trenta valley $\left(46^{\circ} 23^{\prime} \mathrm{N}, 13^{\circ}\right.$ $46^{\prime}$ E), Slovenia. Coll and subm by A Šercelj.

\section{Z-1888. Gračanica}

$180 \pm 110$

Fossil wood (unid. sp), in Spreča $\mathrm{R}$ alluvium, $7 \mathrm{~m}$ depth at Gračanica $\left(44^{\circ} 23^{\prime} \mathrm{N}, 18^{\circ} 46^{\prime} \mathrm{E}\right)$, Bosnia. Coll and subm by H Juruzović, Gračanica. Comment $(\mathrm{HJ})$ : younger than expected (1000 BP).

\section{Z-1775. Plat, B-4}

$12,300 \pm 300$

Fossil wood (unid. sp) from borehole B-4, depth $11.5 \mathrm{~m}$, on slope consisting of clay and Quaternary carbonaceous rocks at Plat near Dubrovnik $\left(42^{\circ} 36^{\prime} \mathrm{N}, 18^{\circ} 14^{\prime} \mathrm{E}\right)$ at $30 \mathrm{~m}$ asl. Coll and subm 1986 by V Jurak, Fac Min, Geol and Petrol Eng, Univ Zagreb. Comment (VJ): geophysical and geological investigations.

\section{GROUNDWATER SAMPLES}

For groundwater samples, model ages (see Introduction) are reported in the Comment. 


\section{Banja Koviljača series}

Thermomineral artesian well, Banja Koviljača spa $\left(44^{\circ} 31^{\prime} \mathrm{N}, 19^{\circ} 10^{\prime} \mathrm{E}\right)$ near Loznica, at $130 \mathrm{~m}$ asl, W Serbia. Coll and subm April 1986 by M Milivojević. Measurements of radioactive and stable isotope content of groundwater. Hydrogeologic study of geothermal potential of aquifer. Age of groundwater was calculated assuming initial groundwater dissolved in organic carbon activity $\left(\mathrm{A}_{\mathrm{o}}\right)=85 \mathrm{pMC}$.

Z-1692. BK-1

Thermal artesian water, depth $80 \mathrm{~m}$, highly mineralized. Tritium activity: $<0.2 \mathrm{~Bq} /$ l. Comment: 21,500 \pm 1200 .

\section{Z-1693. BK-2}

Thermal artesian water, depth $274 \mathrm{~m}$. Tritium activity: $<0.2 \mathrm{~Bq} / \mathrm{l}$. Comment: $21,000 \pm 1150$.

\section{Z-1694. BK-3}

Thermal artesian water, depth $483 \mathrm{~m}$, Triassic karst aquifer. Tritium activity: $<0.4 \pm 0.2 \mathrm{~Bq} / \mathrm{l}$. Comment: 18,600 \pm 1120 .

\section{Z-1691. Vuk Karadžić}

$9.1 \pm 0.5 \mathrm{pMC}$

Thermal artesian water, depth $5.6 \mathrm{~m}$. Tritium activity: $<0.2 \mathrm{~Bq} / \mathrm{l}$. Comment: $18,000 \pm 1050$.

\section{Kostolac series}

Groundwater from aquifer Katanski Brod near Kostolac $\left(44^{\circ} 43^{\prime} \mathrm{N}\right.$, $\left.21^{\circ} 12^{\prime} \mathrm{E}\right)$, E Serbia. Coll and subm March 1987 by M Hadžišehović, Boris Kidrič Inst, Belgrade.

\section{Z-1877. OP 2/1}

Shallow aquifer, ca $4 \mathrm{~m}$ below surface. Expected age: recent water. Tritium activity: $5.4 \pm 0.2 \mathrm{~Bq} / \mathrm{l}$. Comment: modern.

\section{Z-1878. IB-3}

$107.0 \pm 1.4 \mathrm{pMC}$

Shallow aquifer, ca $20 \mathrm{~m}$ below surface. Tritium activity: $6.5 \pm 0.3 \mathrm{~Bq} / \mathrm{l}$. Comment: modern.

\section{Z-1879. B-21/2}

Groundwater, depth $40 \mathrm{~m}$. Tritium activity: $2.6 \pm 0.2 \mathrm{~Bq} / \mathrm{l}$. Comment: $11,460 \pm 990$.

\section{Z-1880. B-21/3}

Groundwater, depth $70 \mathrm{~m}$. Tritium activity: $6.1 \pm 0.3 \mathrm{~Bq} / \mathrm{l}$. Comment: $7460 \pm 970$. 
Z-1882. Malo Lale

Thermal artesian water from karst aquifer Malo Lale $\left(44^{\circ} 23^{\prime} \mathrm{N}, 21^{\circ}\right.$ $25^{\prime}$ E) near Petrovac, E Serbia, depth $160 \mathrm{~m}$. Coll and subm 1987 by $\mathrm{M}$ Hadžišehović. Tritium activity: $<0.2 \mathrm{~Bq} / \mathrm{l}$. Comment: 22,300 \pm 1200 .

\section{Z-1652. Kakanj, IT-1}

$10.1 \pm 0.6 \mathrm{pMC}$

Groundwater, Kakanj ( $44^{\circ} 08^{\prime}$ N, $\left.18^{\circ} 05^{\prime} \mathrm{E}\right), \mathrm{C}$ Bosnia. Coll and subm Jan 1986 by J Slišković, Geoinženjering, Sarajevo. Tritium activity: $1.1 \pm$ $0.2 \mathrm{~Bq} /$ l. Comment: 17,100 1070 .

\section{Z-1838. Ilidža, IB-2}

$15.6 \pm 0.7 \mathrm{pMC}$

Thermal artesian groundwater from borehole in Triassic dolomite, depth 47 to $246 \mathrm{~m}$, highly mineralized. Ilidža spa near Sarajevo $\left(43^{\circ} 50^{\prime} \mathrm{N}\right.$, $18^{\circ} 20^{\prime} \mathrm{E}$ ), $497 \mathrm{~m}$ asl, Bosnia. Coll and subm Feb 1987 by B Djerković, Geoinst, Sarajevo. Hydrogeologic investigation of thermal water resources (DJerković, 1976). Tritium activity: <0.2 Bq/l. Comment: 13,600 \pm 1020 .

\section{Z-1738. Dvorovi}

$5.2 \pm 0.4$ pMC

Thermal water from artesian borehole S-1 at Dvorovi near Bijeljina $\left(44^{\circ} 48^{\prime} \mathrm{N}, 19^{\circ} 16^{\prime} \mathrm{E}\right)$, at $87 \mathrm{~m}$ asl, E Bosnia. Karst aquifer, depth 1200 to $1300 \mathrm{~m}$, weakly mineralized water. Coll and subm Jan 1986 by A Lutvić, Geoinst, Sarajevo. Tritium activity: $0.5 \pm 0.2 \mathrm{~Bq} / \mathrm{l}$. Comment: 22,400 \pm 1140 .

\section{South Banat series}

Study of groundwater in Banat region between Begej channel and Danube R included Deliblatska peščara sand desert. Groundwater in S part of sand desert close to Danube $\mathrm{R}$ is recent, that between Deliblat and Samoš-Velika greda line belongs to Holocene, whereas groundwater in $\mathrm{N}$ part of area is older than 37,000 yr. Coll and subm May 1985 by N Horvatinčić and S Grgić, Rudjer Bošković Inst.

\section{Z-1517. Dolovo}

$28.5 \pm 0.4 \mathrm{pMC}$

Groundwater, pumping station Dolovo ( $\left.44^{\circ} 54^{\prime} \mathrm{N}, 20^{\circ} 53^{\prime} \mathrm{E}\right)$. Tritium activity: <0.2 Bq/1. Comment: $8780 \pm 960$.

\section{Z-1518. Deliblatska peščara 1}

$70.3 \pm 0.7 \mathrm{pMC}$

Groundwater Deliblatska peščara $\left(44^{\circ} 52^{\prime} \mathrm{N}, 21^{\circ} 04^{\prime} \mathrm{E}\right)$. Tritium activity: $<0.2 \mathrm{~Bq} / \mathrm{l}$. Comment: $1530 \pm 950$.

\section{Z-1519. Deliblatska peščara 2}

Groundwater well No. H-1 $1\left(44^{\circ} 51^{\prime} \mathrm{N}, 21^{\circ} 09^{\prime} \mathrm{E}\right)$. Tritium activity: $<0.2 \mathrm{~Bq} /$ l. Comment: $130 \pm 950$ (modern).

\section{Z-1529. Deliblatska peščara 3}

Water from spring Klokot in Deliblatska peščara desert $\left(44^{\circ} 49^{\prime} \mathrm{N}, 21^{\circ}\right.$ $\left.07^{\prime} \mathrm{E}\right)$. Tritium activity: $<0.2 \mathrm{~Bq} / \mathrm{l}$. Comment: $250 \pm 950$ (modern). 
Z-1520. Danube R

$107.15 \pm 0.9 \mathrm{pMC}$

River water near Kovin $\left(44^{\circ} 45^{\prime} \mathrm{N}, 20^{\circ} 59^{\prime} \mathrm{E}\right)$. Tritium activity: $3.6 \pm$ $0.2 \mathrm{~Bq} /$ l. Comment: modern.

Z-1522. Straža

$25.9 \pm 0.5 \mathrm{pMC}$

Groundwater, pumping station Straža $\left(44^{\circ} 57^{\prime} \mathrm{N}, 21^{\circ} 18^{\prime} \mathrm{E}\right)$. Tritium activity: $<0.2 \mathrm{~Bq} /$ l. Comment: $9550 \pm 960$.

Z-1523. Dupljaja

$45.5 \pm 0.5 \mathrm{pMC}$

Groundwater from artesian well at Dupljaja $\left(45^{\circ} 55^{\prime} \mathrm{N}, 21^{\circ} 17^{\prime} \mathrm{E}\right)$. Tritium activity: $<0.2 \mathrm{~Bq} / \mathrm{l}$. Comment: $5020 \pm 950$.

Z-1524. Šušara

$55.0 \pm 0.5 \mathrm{pMC}$

Groundwater from pumping station at Šušara $\left(44^{\circ} 56^{\prime} \mathrm{N}, 21^{\circ} 07^{\prime} \mathrm{E}\right)$, $200 \mathrm{~m}$ depth. Tritium activity: $<0.2 \mathrm{~Bq} / \mathrm{l}$. Comment: $3500 \pm 950$.

Z-1525. Vladimirovac

$32.5 \pm 0.5 \mathrm{pMC}$

Groundwater from pumping water at Vladimirovac $\left(45^{\circ} 02^{\prime} \mathrm{N}, 20^{\circ} 52^{\prime}\right.$ E). Tritium activity: $<0.2 \mathrm{~Bq} / \mathrm{l}$. Comment: $7720 \pm 960$.

Z-1526. Alibunar

$43.1 \pm 0.2 \mathrm{pMC}$

Artesian water at Alibunar $\left(45^{\circ} 05^{\prime} \mathrm{N}, 20^{\circ} 58^{\prime} \mathrm{E}\right)$. Tritium activity: $<0.2$ $\mathrm{Bq} / \mathrm{l}$. Comment: $5460 \pm 950$.

\section{Z-1527. Potporanj}

$36.5 \pm 0.5 \mathrm{pMC}$

Groundwater from well at Potporanj $\left(45^{\circ} 05^{\prime} \mathrm{N}, 21^{\circ} 15^{\prime} \mathrm{E}\right)$. Tritium activity: $<0.2 \mathrm{~Bq} / \mathrm{l}$. Comment: $6790 \pm 960$.

\section{Z-1528. Gaj}

$70.6 \pm 0.6 \mathrm{pMC}$

Groundwater from well at Gaj $\left(44^{\circ} 47^{\prime} \mathrm{N}, 21^{\circ} 04^{\prime} \mathrm{E}\right)$. Tritium activity: $<0.2 \mathrm{~Bq} / \mathrm{l}$. Comment: $1490 \pm 950$.

Z-1531. Samoš

$10.6 \pm 0.4 \mathrm{pMC}$

Groundwater, pumping station at Samoš $\left(45^{\circ} 12^{\prime} \mathrm{N}, 20^{\circ} 46^{\prime} \mathrm{E}\right)$. Tritium activity: $<0.2 \mathrm{~Bq} /$ l. Comment: 16,720 \pm 1000 .

Z-1532. Ilandža

$16.3 \pm 0.4 \mathrm{pMC}$

Artesian water at Ilandža $\left(45^{\circ} 10^{\prime} \mathrm{N}, 20^{\circ} 55^{\prime} \mathrm{E}\right)$. Tritium activity: $<0.2$ $\mathrm{Bq} / \mathrm{l}$. Comment: $13,270 \pm 970$.

Z-1533. Kovačica

$13.7 \pm 0.4 \mathrm{pMC}$

Tap-water, sugar refinery water supply, Kovačica $\left(45^{\circ} 07^{\prime} \mathrm{N}, 20^{\circ} 37^{\prime} \mathrm{E}\right)$. Tritium activity: $<0.2 \mathrm{~Bq} / \mathrm{l}$. Comment: 14,660 \pm 980.

Z-1534. Zrenjanin

Groundwater, pumping station in Zrenjanin $\left(45^{\circ} 23^{\prime} \mathrm{N}, 20^{\circ} 24^{\prime} \mathrm{E}\right)$. Tritium activity: $<0.2 \mathrm{~Bq} / \mathrm{l}$. Comment: 25,200 \pm 1290 . 


\section{Z-1535. Žitište}

$$
1.1 \pm 0.3 \mathrm{pMC}
$$

Tap-water, Žitište $\left(45^{\circ} 29^{\prime} \mathrm{N}, 20^{\circ} 34^{\prime} \mathrm{E}\right)$. Tritium activity: $<0.2 \mathrm{~Bq} / \mathrm{l}$. Comment: $35,000 \pm 2500$.

\section{Z-1536. Velika greda}

$11.0 \pm 0.4 \mathrm{pMC}$

Artesian well at Velika greda $\left(45^{\circ} 15^{\prime} \mathrm{N}, 21^{\circ} 02^{\prime} \mathrm{E}\right)$. Tritium activity: $<0.2 \mathrm{~Bq} /$ l. Comment: 16,400 \pm 990 .

\section{Z-1537. Sečanj}

Artesian well at Sečanj $\left(45^{\circ} 22^{\prime} \mathrm{N}, 20^{\circ} 46^{\prime} \mathrm{E}\right)$. Tritium activity: $<0.2$ $\mathrm{Bq} / \mathrm{l}$. Comment: $>37,000$.

\section{Z-1538. Krajišnik}

Groundwater from pumping station at Krajišnik $\left(45^{\circ} 27^{\prime} \mathrm{N}, 20^{\circ} 44^{\prime} \mathrm{E}\right)$. Tritium activity: $<0.2 \mathrm{~Bq} / \mathrm{l}$. Comment: $27,000 \pm 1300$.

\section{Z-1946. Danube R}

$103.2 \pm 1.4 \mathrm{pMC}$

River water near Dubovac $\left(44^{\circ} 48^{\prime} \mathrm{N}, 18^{\circ} 53^{\prime} \mathrm{E}\right)$. Coll and subm October 1987 by B Orlić. Tritium activity: $3.8 \pm 0.2 \mathrm{~Bq} / \mathrm{l}$. Comment: modern.

Z-1539. Tomaševac

$3.2 \pm 0.4$ pMC

Artesian well at Tomaševac $\left(45^{\circ} 16^{\prime} \mathrm{N}, 20^{\circ} 37^{\prime} \mathrm{E}\right)$. Tritium activity: $<0.2 \mathrm{~Bq} /$ l. Comment: $26,300 \pm 1400$.

\section{CALCAREOUS SEDIMENTS}

\section{Z-1298. Obrovac Tunnel}

$>37,000$

Crystalline speleothem from cavern in tunnel of hydroelectric power plant on Zrmanja R near Obrovac $\left(44^{\circ} 11^{\prime} \mathrm{N}, 15^{\circ} 41^{\prime} \mathrm{E}\right)$ Dalmatia, S Croatia. Coll and subm 1982 by S Božičević, Inst Geol, Zagreb.

\section{Z-1350. Švica Tunnel}

$84.5 \pm 0.6 \mathrm{pMC}$

Calcareous tufa deposited on outer rim of discharge tunnel of small hydroelectric power plant at Švica $\left(44^{\circ} 52^{\prime} \mathrm{N}, 15^{\circ} 10^{\prime} \mathrm{E}\right)$ Lika, Croatia. Plant in operation from 1939 to 1963 . Coll and subm 1984 by D Srdoč. Comment (DS): tufa deposited before bomb-test contamination.

\section{Perinka ponor series}

Wood branches and timber partially encrusted with calcareous deposits from Perinka ponor (swallow hole) at $50 \mathrm{~m}$ depth at Donja Švica near Otočac (44 $\left.52^{\prime} \mathrm{N}, 15^{\circ} 10^{\prime} \mathrm{E}\right)$, Lika, central Croatia. Coll and subm 1987 by $\mathrm{S}$ Božičević, Geol Inst Zagreb. Comments (SB): dates phases of tufa precipitation. (DS): calcareous deposits from pre-bomb test era on two wooden samples enable determination of the initial activity $A_{o}$ of groundwater and calcareous deposits.

Z-1905. No. 1

$99.9 \pm 1.5 \mathrm{pMC}$

Wooden beam at entrance of hole. 
Z-1906. No. 2

$68.8 \pm 1.1 \mathrm{pMC}$

Inner part of calcareous deposit, 35m depth.

Z-1907. No. 3

$67.7 \pm 1.1 \mathrm{pMC}$

Calcareous deposit on wooden beam (Z-1908), 40m depth. Comment (DS): ratio of contemporaneous wood and tufa ${ }^{14} \mathrm{C}$ activities $\mathrm{A}_{\mathrm{o}}=0.69$.

Z-1908. No. 4

$97.8 \pm 1.4 \mathrm{pMC}$

Wooden beam coated with calcite layer, $40 \mathrm{~m}$ depth.

Z-1909. No. 5

$68.7 \pm 1.1 \mathrm{pMC}$

Tufa coating on twig (Z-1910), 50m depth. Comment (DS): ratio of contemporaneous wood and tufa ${ }^{14} \mathrm{C}$ activities $\mathrm{A}_{\mathrm{o}}=0.68$.

Z-1910. No. 6.

$101.4 \pm 1.4 \mathrm{pMC}$

Twig with calcite coating, $50 \mathrm{~m}$ depth.

\section{Kapsia cave series}

Two stalagmites from Kapsia cave with sink hole $\left(37^{\circ} 38^{\prime} \mathrm{N}, 22^{\circ} 24^{\prime} \mathrm{E}\right)$, at $630 \mathrm{~m}$ asl, Peleponnesus, S Greece. Coll and subm 1985 by R Gospodarič, Slov Acad Sci \& Arts, Postojna. Comment (RG): dated to determine period of Holocene flood.

Z-1604. K-2, No. 1

$53.6 \pm 0.6 \mathrm{pMC}$

Core of rust-colored stalagmite. Comment: $3700 \pm 950$.

Z-1606. K-2, No. 2a

$54.9 \pm 0.5 \mathrm{pMC}$

Middle part of same stalagmite. Comment: $3510 \pm 950$.

Z-1608. K-2, No. 3

$55.6 \pm 0.5 \mathrm{pMC}$ 950.

Top of same stalagmite, 1.5 to $3.0 \mathrm{~cm}$ below surface. Comment: $3410 \pm$

Z-1683. K-1a

$88.7 \pm 0.8 \mathrm{pMC}$

Upper part of stalagmite. Comment: modern.

PEAT AND SOIL SAMPLES

\section{Lovrenško barje series}

Peat samples from borehole, Lovrenško barje peat bog, Pohorje Mt, $\left(46^{\circ} 29^{\prime} \mathrm{N}, 13^{\circ} 00^{\prime} \mathrm{E}\right)$, at 1300 asl, N Slovenia. Coll and subm 1986 by A Sercelj, Slov Acad Sci \& Arts, Ljubljana. Dates help determine forest vegetation development (Culiberg, 1986). Comment: very good stratigraphic correlation with Z-1157, 130 to $140 \mathrm{~cm}$ and Z-1158, 200 to $220 \mathrm{~cm}$ (R, 1984, v 26 , no. 3 , p 454).

Z-1773. Lovrenško barje, No. 1

$1620 \pm 130$

40 to $60 \mathrm{~cm}$ depth. 
230 to $250 \mathrm{~cm}$ depth.

\section{Ribniško barje series}

Peat from bog Ribniško barje $\left(46^{\circ} 30^{\prime} \mathrm{N}, 12^{\circ} 56^{\prime} \mathrm{E}\right)$, at $1550 \mathrm{~m}$ asl, Pohorje Mt, N Slovenia. Coll and subm 1986 by A Šercelj. Dates help determine forest vegetation development periods (Culiberg, 1986). Corresponds to earlier reported results, $c f \mathrm{Z}-1365,-1366(\mathrm{R}, 1987, \mathrm{v} 29$, no. $1, \mathrm{p}$ 142).

Z-1780. Ribniško barje, No. 1

$$
2790 \pm 130
$$

Peat, depth 40 to $60 \mathrm{~cm}$. Expected age, $3000 \mathrm{yr}$ BP.

\section{Z-1781. Ribniško barje, No. 2}

Peat, depth $250 \mathrm{~cm}$. Expected age, $5000 \mathrm{yr}$ BP.

\section{Z-1947. Morijeve luže}

$4130 \pm 150$

Peat from Morijeve luže bog near Dravograd $\left(46^{\circ} 39^{\prime} \mathrm{N}, 15^{\circ} 5^{\prime} \mathrm{E}\right)$, at $1000 \mathrm{~m}$ asl, N Slovenia. Coll and subm 1987 by A Šercelj. Systematic palynol investigation of Holocene vegetation in Slovenia. Comment (AS̆): expected age: Holocene.

\section{Malo polje series}

Peat and gyttja from moor in Malo polje $\left(46^{\circ} 21^{\prime} \mathrm{N}, 13^{\circ} 51^{\prime} \mathrm{E}\right)$ at $1600 \mathrm{~m}$ asl, Triglav Mt, Julian Alps, NW Slovenia. Coll and subm 1987 by A Sercelj. Systematic palynol investigation of Holocene vegetation in Slovenia, NW Yugoslavia (Šercelj, 1965).

\section{Z-1922. Malo polje, No. 1}

$$
5720 \pm 170
$$

Peat, 70 to $100 \mathrm{~cm}$ depth.

\section{Z-1923. Malo polje, No. 2}

$6500 \pm 180$

Gyttja, 100 to $120 \mathrm{~cm}$ depth.

\section{Z-1951. N Adriatic shelf}

$$
9030 \pm 270
$$

Peat from sediment core $80 \mathrm{~cm}$ below sea bottom, N Adriatic Sea $\left(44^{\circ}\right.$ $50^{\prime} \mathrm{N}, 13^{\circ} 30^{\prime}$ E) $40 \mathrm{~km}$ W of Pula, Istria, W Croatia. Coll and subm 1987 by S Puškarić, CIM Rudjer Bošković Inst, Rovinj. Comment (SP): good stratigraphic correlations with Z-713 (R, 1981, v 23, no. 3, p 415).

\section{Z-1609. Sajan E-7869}

$9470 \pm 180$

Organic fraction of soil (chernozem) from K-22-Sn core, depth 1.8 to $1.9 \mathrm{~m}$. Sajan $\left(45^{\circ} 51^{\prime} \mathrm{N}, 20^{\circ} 16^{\prime} \mathrm{E}\right)$ near Kikinda, N Banat, at $82 \mathrm{~m}$ asl. Coll and subm 1985 by D Koprivica, Geoinst Belgrade. Dated for drafting geologic map of AP Vojvodina, NE Yugoslavia. Comment (DK): expected age: Holocene. 
Organic fraction of soil from sandy soil profile, depth 0.4 to $0.7 \mathrm{~m}$, at Bočar $\left(45^{\circ} 46^{\prime} \mathrm{N}, 20^{\circ} 16^{\prime} \mathrm{E}\right)$ near Kikinda, N Banat, 93m asl. Coll and subm 1986 by D Koprivica. Dated for drafting of geologic map of Yugoslavia. Comment (DK): expected age: Holocene.

Z-1612. Crna bara E-7963

$2530 \pm 150$

Organic fraction of soil from sandy soil profile, depth 0.35 to $0.45 \mathrm{~m}$, at Crna bara ( $\left.45^{\circ} 59^{\prime} \mathrm{N}, 20^{\circ} 16^{\prime} \mathrm{E}\right)$, near Kikinda, N Banat, at $82 \mathrm{~m}$ asl. Coll and subm 1985 by D Koprivica. Comment (DK): expected age: Holocene.

\section{Bogojevo series}

Organic fraction of soil from profile and fossil wood from borehole at Bogojevo ( $45^{\circ} 32^{\prime} \mathrm{N}, 19^{\circ} 07^{\prime} \mathrm{E}$ ), S Bačka, AP Vojvodina, near Danube R. Geotectonic investigations in NE Serbia. Coll and subm by N Krstić, Geoinst, Belgrade.

Z-1676.

$7750 \pm 150$

Depth 4.8 to $4.9 \mathrm{~m}$.

Z-1677.

$34,200+3200$

Depth 23.85 to $24.00 \mathrm{~m}$.

Z-1678.

Depth 24.5 to $24.65 \mathrm{~m}$.

Z-1679.

Depth 35.8 to $36.0 \mathrm{~m}$.

Z-1627. LS-5

$9270 \pm 180$

Fossil wood (unid. sp) from borehole LS-5, Danube R sandy alluvium, depth 16.3 to $17.0 \mathrm{~m}$. Comment (NK): expected age based on paleologic analyses: Holocene.

\section{Prevlaka series}

Organic clay and peat from borehole, Prevlaka near Zagreb $\left(45^{\circ} 40^{\prime} \mathrm{N}\right.$, $\left.16^{\circ} 16^{\prime} \mathrm{E}\right) \mathrm{W}$ Croatia. Coll and subm 1984 by E Prohić, Geol Inst, Zagreb. Geotectonic investigations.

Z-1741. S-5

$29,000 \pm 1100$ BP.

Organic clay, depth 15.4 to $16.0 \mathrm{~m}$. Comment (EP): expected age 30,000

Z-1756. S-10 $>\mathbf{3 7 , 0 0 0}$

Peat, depth 26.2 to $27.0 \mathrm{~cm}$. Comment (EP): expected age: 40,000 BP. 


\section{Lipar series}

Soil carbonate from Lipar ( $\left.45^{\circ} 42^{\prime} \mathrm{N}, 19^{\circ} 33^{\prime} \mathrm{E}\right)$, Bačka loess plateau in NE Yugoslavia. Coll and subm 1987 by M Galečić, Geoinst, Belgrade. Dating of loess formation for drafting of geologic map of Yugoslavia.

Z-1893. D-91322

$50.1 \pm 0.9$ pMC

Depth $1.2 \mathrm{~m}$. Humic loess sediment (chernozem) assoc with roots and shell fragments. Comment (MG): expected age: Würm-Holocene.

Z-1892. D-91321

$14.6 \pm 0.7 \mathrm{pMC}$

Humic loess assoc with shell and fauna fragments, depth $1.8 \mathrm{~m}$. Comment (MG): expected age: Würm. General Comment: initial activity of soil carbonate is not known.

Z-1895. Banatsko Karadordevo D-87486

$\mathbf{1 5 , 5 3 0} \pm \mathbf{3 8 0}$

Macrofossil remains in sediment, Banatsko Karadordevo $\left(45^{\circ} 34^{\prime} \mathrm{N}\right.$, $20^{\circ} 35^{\prime} \mathrm{E}$ ), Banat, depth $3.5 \mathrm{~m}$. Coll and subm 1984 by S Žolnaj, Geoinst, Belgrade. Comment (SŽ): expected age: Würm; dates terraces of Tisa and Tamiš Rivers.

\section{Z-1896. Čestereg D-87485}

$11,930 \pm 250$

Macrofossil remains in sandy river sediment at Čestereg $\left(45^{\circ} 34^{\prime} \mathrm{N}, 20^{\circ}\right.$ $32^{\prime}$ E), Banat, depth $4 \mathrm{~m}$. Coll and subm 1987 by S Žolnaj. Comment (SŽ): expected age: Würm.

\section{Z-1628. Otok, Vi-85, 3882}

$7830 \pm 120$

Calcareous concretions from clayey silt, near Otok $\left(45^{\circ} 09^{\prime} \mathrm{N}, 18^{\circ} 53^{\prime}\right.$ E), Slavonia, E Croatia. Coll and subm 1985 by M Brkić, Geol Inst, Zagreb. Comment (MB): dated for drafting of geologic map of Slavonia.

\section{Vinkovci series}

Clayey silt assoc with charred wood fragments and silty clay from pit at Privlaka ( $45^{\circ} 12^{\prime} \mathrm{N}, 18^{\circ} 51^{\prime} \mathrm{E}$ ), near Vinkovci, E Croatia. Coll and subm 1985 by M Brkić.

Z-1629. 5422/4

$2100 \pm 90$

Clayey silt assoc with charred wood fragments, depth $0.5 \mathrm{~m}$.

Z-1630. 5422/1

$17,900 \pm 460$

Silty clay with organic detritus, Žanića Krivina, depth $6 \mathrm{~m}$.

\section{Z-1640. Gornja Gorevnica T-3060/II}

$4.1 \pm 0.3 \mathrm{pMC}$

Loess from brickyard Gornja Gorevnica near Sombor $\left(45^{\circ} 48^{\prime} \mathrm{N}, 19^{\circ}\right.$

$27^{\prime}$ E) at $104 \mathrm{~m}$ asl, AP Vojvodina. Coll and subm 1985 by S Trifunović, Geol Inst, Belgrade. Age close to limit for loess deposits. Initial activity of carbonates is unknown. Comment: $24,350 \pm 1120$. 
Land snail shells from sediment, Profile IX, partly contaminated with carbonate concretions. Draž near Batina ( $45^{\circ} 50^{\prime} \mathrm{N}, 18^{\circ} 46^{\prime} \mathrm{E}$ ), Baranja, E Croatia, at $190 \mathrm{~m}$ asl. Coll and subm 1986 by M Pikija, Geol Inst, Zagreb. Comment (MP): dated for drafting of geologic map of Yugoslavia.

\section{Branjina series}

Calcareous concretions and snail shells from sediment, Branjina $\left(45^{\circ}\right.$ $50^{\prime} \mathrm{N}, 18^{\circ} 42^{\prime} \mathrm{E}$ ), Baranja, E Croatia, at $130 \mathrm{~m}$ asl. Coll and subm 1986 by $\mathrm{M}$ Pikija. Comment (MP): expected age: Pleistocene. Dated for drafting of geologic map of Yugoslavia.

Z-1644. MO-231(K)

Calcareous concretions. Comment: $22,600 \pm 1350$.

\section{Z-1649. MO-231(P)}

Land snail shells.

General Comment: calcareous concretions show younger age, indicating carbonate-rich freshwater penetration.

\section{Kneževo series}

Calcareous concretions and snail shells from sediment in brickyard, Kneževo $\left(45^{\circ} 51^{\prime} \mathrm{N}, 18^{\circ} 39^{\prime} \mathrm{E}\right), 197 \mathrm{~m}$ asl, Baranja, E Croatia. Coll and subm 1986 by M Pikija. Comment (MP): expected age: Pleistocene. Dated for drafting of geologic map of Yugoslavia.

\section{Z-1647. MO-473(K)}

$$
76.5 \pm 0.8 \mathrm{pMC}
$$

Calcareous concretions. Comment: $850 \pm 950$.

Z-1648. MO-473(P)

$$
16,000 \pm 280
$$

Land snail shells.

Z-1651. Zmajevac

$$
\mathbf{2 0 , 4 0 0} \pm \mathbf{4 6 0}
$$

Land snail shells from sediment, Zmajevac brickyard $\left(45^{\circ} 48^{\prime} \mathrm{N}, 18^{\circ}\right.$ $48^{\prime} \mathrm{E}$ ), at $130 \mathrm{~m}$ asl, Baranja, E Croatia. Coll and subm 1986 by M Pikija. Comment (MP): expected age: Pleistocene. Dated for drafting of geologic map of Yugoslavia.

\section{REFERENCES}

Culiberg, M, 1986, Palynological investigations of two peat-bogs, Lovrenško and Ribniško barje from the Pohorje Mts: Biol Vestn, v 34, p 1-14.

Djerković, B, 1976, Podzemni karstni oblici kao prirodni rezervoari termalne vode: Geol glasnik, v 21, p 231-238.

Gimbutas, M, 1980, The Kurgan Wave 2 (c 3400-3200 BC) into Europe and the following transformation of culture: Jour Indo-European Studies, v 8, nos. 3-4, p 273-315.

Horvatinčić, N, Obelić, B, Srdoč, D, Durman, A, Benkö, L and Sliepčević, A, in press, Radiocarbon and TL dating of the Eneolithic site Vučedol in East Croatia, Yugoslavia: Pact.

Krivic, K, 1985, Los tudi na Soriški planini: Loški razgledi, v 32, p 93-97. 
Obelić, B, 1980, Computer analysis and interpretation of radiocarbon data: Fizika, v 12, supp 2, p $139-161$.

Obelić, B and Planinić, J, 1977, Computer processing of radiocarbon and tritium data, in Povinec, $\mathrm{P}$ and Usačev, $\mathrm{S}$, eds, Internatl conf on low-radioactivity measurement and applications, Proc: The High Tatras, Slovenske pedagogicke nakladatelstvo, Bratislava, p 117 120.

Srdoč, D, Breyer, B and Sliepčević, A, 1971, Rudjer Bošković Institute radiocarbon measurements I: Radiocarbon, v 13, no. 1, p 135-140.

Srdoč, D and Horvatinčić, N, 1986, Radiocarbon dating of Liber linteus Zagrabiensis: Vjesnik Arheol muz Zagreb, ser 3, v XIX, p 83-98. in press, Radiocarbon dating of an Etruscan linen book: Pact.

Srdoč, D, Krajcar Bronić, I, Horvatinčić, N and Obelić, B, 1986, Increase of ${ }^{14} \mathrm{C}$ activity of dissolved inorganic carbon along a river course, in Stuiver, M and Kra, R S, eds, Internat ${ }^{14} \mathrm{C}$ conf, 12th, Proc: Radiocarbon, v 28, no. 2A, p 515-521.

Srdoč, D, Obelić, B, Horvatinčić, N, Krajcar, I and Sliepčević, A, 1984, Rudjer Bošković Institute radiocarbon measurements VIII: Radiocarbon, v 26, no. 3, p 449-460.

Srdoč, D, Obelić, B, Sliepčević, A, Krajcar Bronić, I and Horvatinčić, N, 1987, Rudjer Bošković Institute radiocarbon measurements X: Radiocarbon, v 29, no. 1, p 135-147.

Srdoč, D, Sliepčević, A, Obelić, B and Horvatinčić, N, 1979, Rudjer Bošković Institute radiocarbon measurements V: Radiocarbon, v 21, no. 1, p 131-137.

Stuiver, M, and Polach, H A, 1977, Discussion: Reporting of ${ }^{14} \mathrm{C}$ data: Radiocarbon, v 19, no. 3 , p 355-363.

Sercelj, A, 1965, Paleofloristic research in the Triglav mountains: Razprave SAZU, v VIII, p 473-498. 